\title{
Thruster-AUV's Motion Adjustment Method Based on Fuzzy Control
}

\author{
Lei Wan ${ }^{1}$, Yinghao Zhang ${ }^{1, a}$, Yushan Sun ${ }^{1}$, and Yueming $\mathrm{Li}^{1}$ \\ ${ }^{1}$ State Key Laboratory of Autonomous Underwater Vehicle, Herbin Engineering University, China \\ azhangyinghao555@126.com
}

Keywords: AUV, thruster-AUV, motion adjustment, fuzzy control, switching strategy

\begin{abstract}
An autonomous underwater vehicle's(AUV) working condition is usually complex, which means that it's hard to build the AUV's dynamic model and it's challenging to find a good method to adjust its motion accurately in that environment. In order to solve that problem, one motion control method based on fuzzy control is offered, which has the less dependence on AUV's model and can express designer's experience easily. One depth control simulation is made to test the control method on one thruster-AUV. The simulation results show that the motion adjustment method based on fuzzy control is feasibility and has a large control area. This method offers a new way to solve the AUV's motion adjustment.
\end{abstract}

\section{Introduction}

AUV's dynamical system is usually nonlinear and time-varying, which is mainly caused by the mutual coupling of the movement from different freedoms and the complexity of working environment [1][2]. Taking that into consideration, it's very difficult to establish an accurate dynamic model of AUV. Therefore, the researchers in this field try to consider the modern control methods which don't depend on the mathematical model too much, for example, fuzzy control. Fuzzy control is a method with strong subjective opinion. The design of the parameters has a direct impact on the control effects, such as overshoot, oscillation and divergence. The complex of the parameters' adjustment limits the wide use of fuzzy control[3]. Even though, some researchers try to use it into AUV's field. In this paper, we try to use the fuzzy control on a thruster-AUV's motion adjustment.

\section{Fuzzy control}

In 1965, L.A.Zadeh brought fuzzy logic into control field first [4] and gave the theoretical basis of fuzzy reasoning. Since then, the application of fuzzy logic has begun its development in control field. The controller based on fuzzy theory includes the following three processes, they are the fuzzy input, fuzzy reasoning and defuzzification, shown as Fig.1.

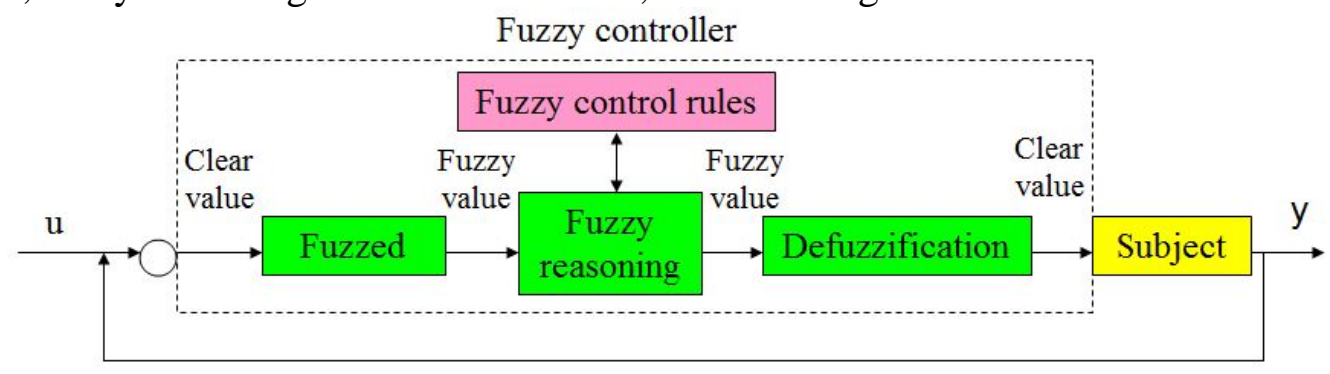

Fig.1. Information frame of fuzzy controller

Corresponding to the union, intersection, complement in classical geometry, fuzzy control method has similar operations and there are linguistic variables in it. Linguistic variables are used to describe the inputs and outputs with negative big (NB), negative medium (NM), negative small (NS), zero (ZO), positive small (PS), positive medium (PM), positive big (PB) and so on. These linguistic variables can be changed by membership functions between fuzzy values and numbers. 
Fuzzy statistics, instance-method and expertise-method are usually used to confirm the membership functions [5]. The reasoning of fuzzy control can be shown by the fuzzy control rulers, show as Table.1. In Table.1, U, E and EC mean input, deviation and deviation variation rate separately.

Table.1. Fuzzy control rulers

\begin{tabular}{|c|c|c|c|c|c|c|c|}
\hline $\mathrm{U}$ & NB & NM & NS & $\mathrm{ZO}$ & PS & PM & PB \\
\hline NB & PB & PB & PB & $\mathrm{PB}$ & PM & $\mathrm{ZO}$ & $\mathrm{ZO}$ \\
\hline NM & PB & PB & PB & PB & PM & $\mathrm{ZO}$ & $\mathrm{ZO}$ \\
\hline NS & PM & PM & PM & $\mathrm{PM}$ & $\mathrm{ZO}$ & NS & NS \\
\hline $\mathrm{ZO}$ & PM & PM & PS & $\mathrm{ZO}$ & NS & NM & NM \\
\hline PS & PS & PS & $\mathrm{ZO}$ & NM & NM & NM & NM \\
\hline $\mathrm{PM}$ & $\mathrm{ZO}$ & $\mathrm{ZO}$ & NM & $\mathrm{NB}$ & NB & NB & $\mathrm{NB}$ \\
\hline PB & $\mathrm{ZO}$ & $\mathrm{ZO}$ & NM & $\mathrm{NB}$ & NB & NB & NB \\
\hline
\end{tabular}

The output of the fuzzy reasoning is a fuzzy set, however, fuzzy controller's outputs must be certain value. This is related to the defuzzification of the results. There are several ways to do that, such as bisector-method, the method of maximum membership function and centroid-method [6].

\section{Motion control based on fuzzy theory}

AUV exists the movement in six degrees of freedom in ocean, they are longitudinal movement, sway, heave, pitch, roll and yaw. In this paper, we use the depth adjustment as an example to show the use of fuzzy control in AUV's motion adjustment. In order to simplify the adjustment of fuzzy control, we decide to use a combination controller of fuzzy control and PID. Fig.2. shows the information frame in depth control.

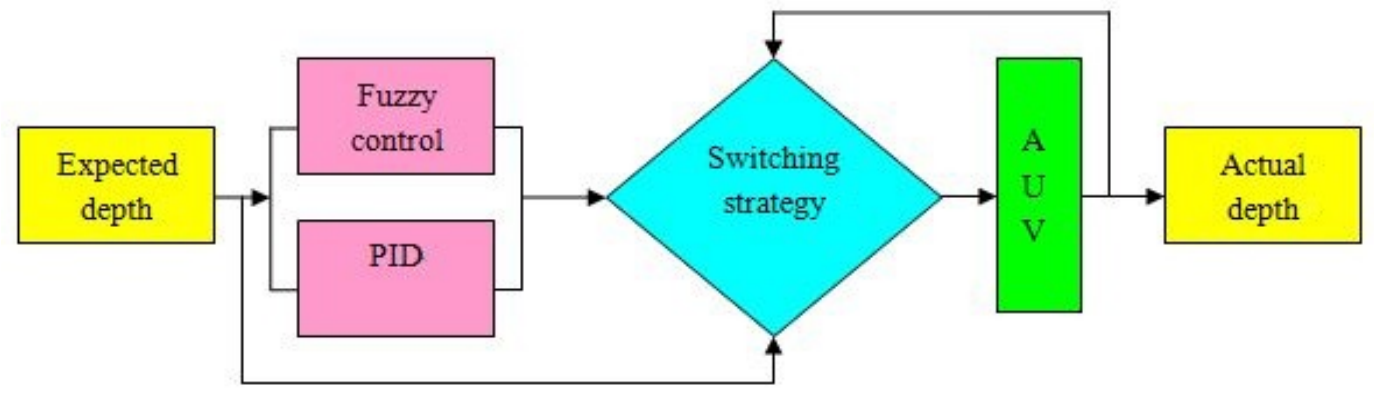

Fig.2. Information frame in depth control based on fuzzy control

\section{Simulation and results}

Simulation subject. The simulation subject is called thruster-AUV, which isn't equipped with wings and rudders. Its motion adjustment is all depended on the thrusters. The arrangement of the thrusters is shown in Fig.3.

This AUV's corresponding parameters are $Z_{w|w|}{ }^{\prime}=-0.224, Z_{w w}{ }^{\prime}=0.285, Z_{\dot{w}}{ }^{\prime}=0.0745$ and $M=1031 \mathrm{~kg}$. The transfer function between depth and vertical force is shown as (1).

$H_{z}(s)=\frac{z(s)}{F(s)}=\frac{1}{s} H_{w}(s)=\frac{1}{887.491 s^{2}+130.833 s}$ 


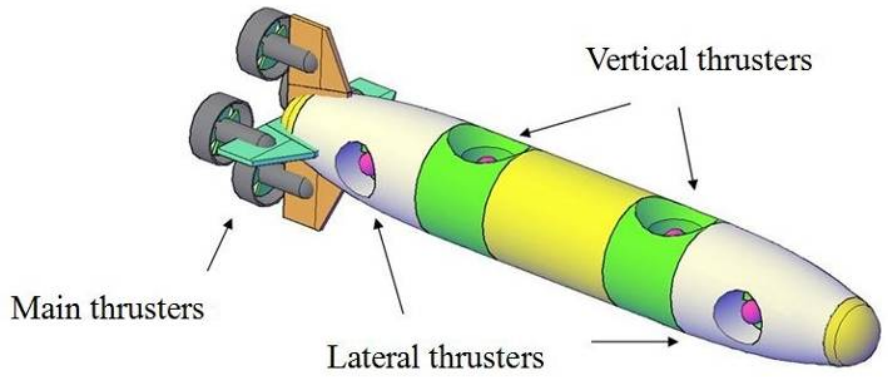

Fig.3. Thruster-AUV

Fuzzy control simulation. In the simulation, we set the switching strategy as $0.5 \mathrm{~m}$, which means that when the absolute value of deviation between expected depth and actual depth is more than $0.5 \mathrm{~m}$, we choose fuzzy control, on the contrary, we choose PID. The membership functions of E, EC and U are shown in Fig.4(a), Fig.4(b) and Fig.4(c). Using the fuzzy control ruler shown in Table 1, the surface of fuzzy control rulers is shown in Fig.4(d).

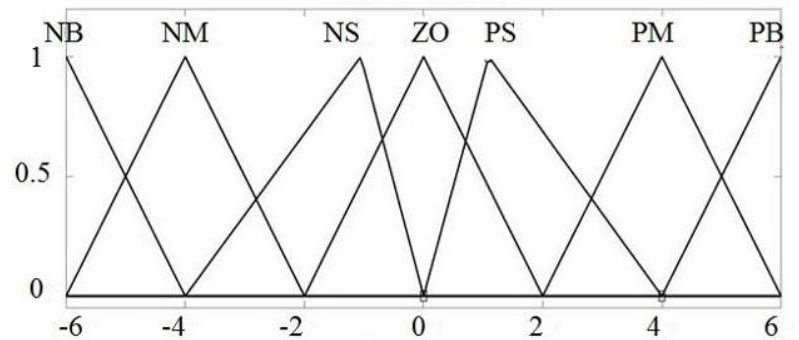

(a) Input variable $\mathrm{E}$

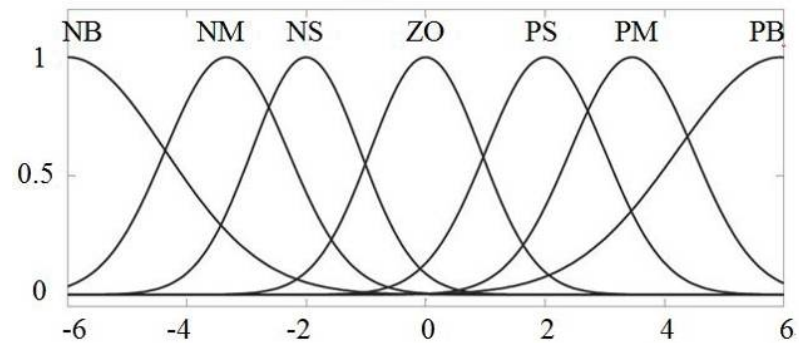

(c) Output variable U

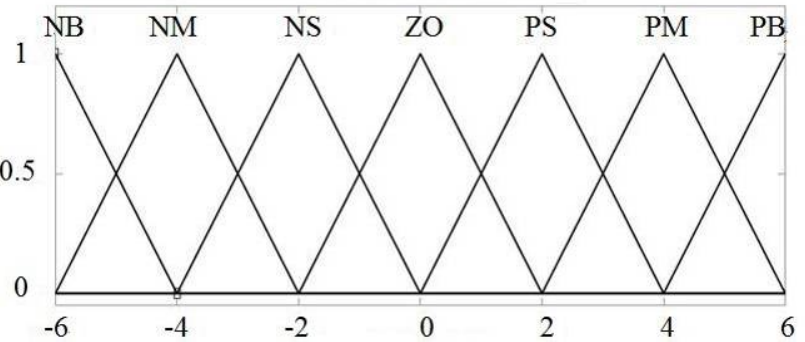

(b) Input variable EC

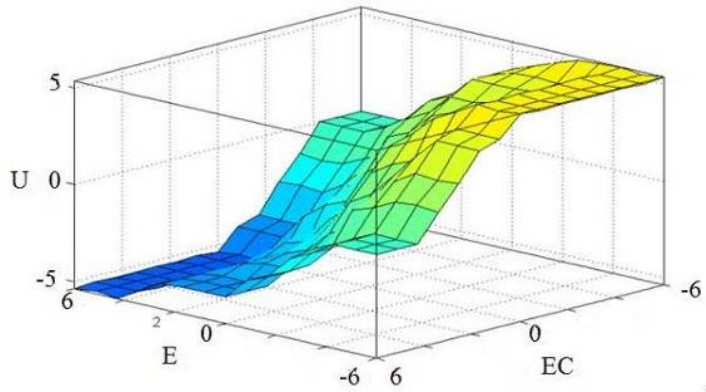

(d) Surface of fuzzy control rulers

Fig.4. Membership function plots and surface of fuzzy control rulers

We choose centroid-method to achieve defuzzification and set PID's parameters as $K_{P}=10$, $K_{I}=0.5$ and $K_{D}=5$.

Simulation results and analysis. We simulate the depth control of $5 \mathrm{~m}, 10 \mathrm{~m}, 50 \mathrm{~m}$ and $100 \mathrm{~m}$ separately, and the simulation results are shown in Fig.5. The simulation results show that fuzzy-PID has a large control area. The use of fuzzy control reduces the dependency on the thruster-AUV's mathematical model and the combination of fuzzy control and PID control reduce the complex of the adjustment of fuzzy control's parameters.

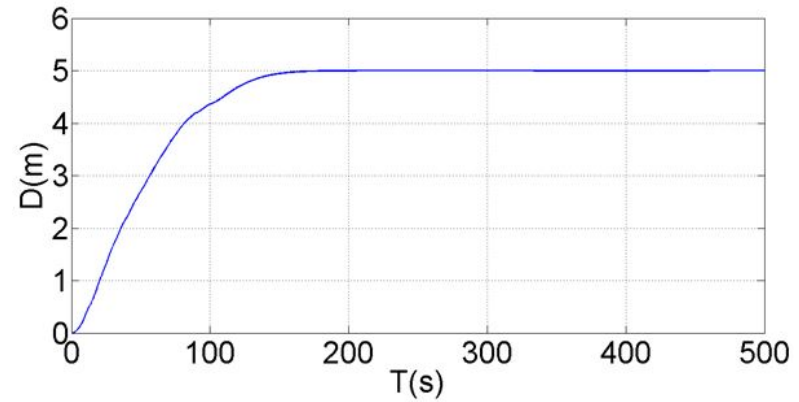

(a) Depth $5 \mathrm{~m}$

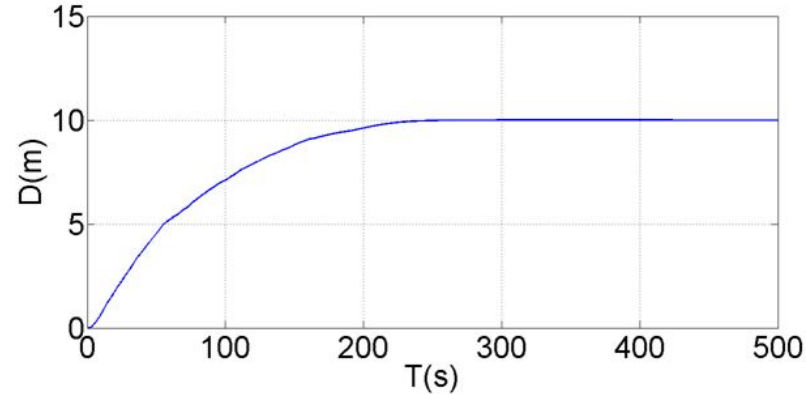

(b) Depth $10 \mathrm{~m}$ 


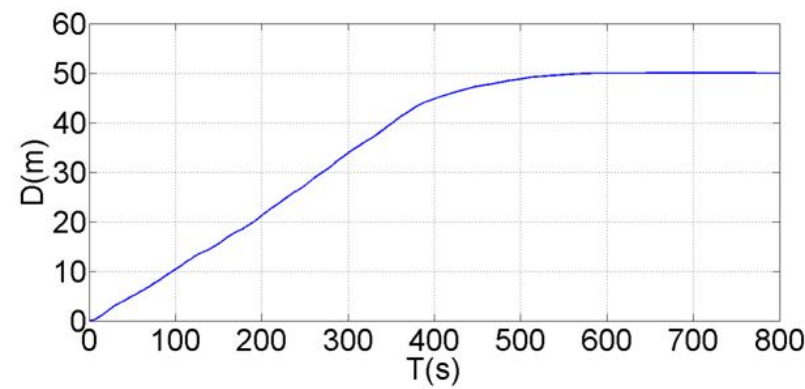

(c) Depth 50m

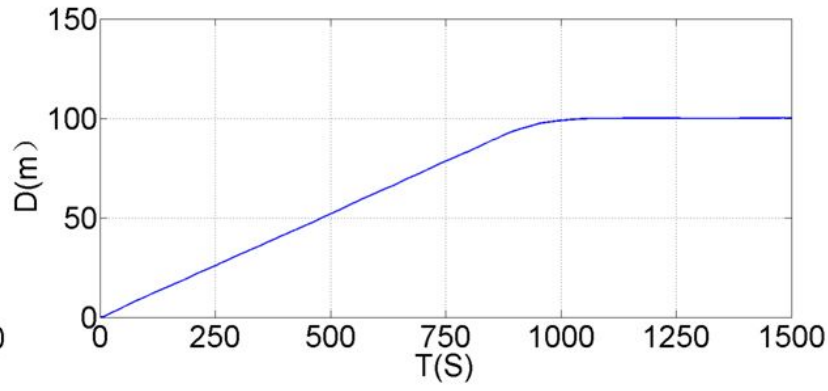

(d) Depth $100 \mathrm{~m}$

Fig.5. Simulation results

\section{Conclusions}

From the results, it can be seen that fuzzy control can express designer's strategy and experience easily. Thanks to the fuzzy control rulers, this method can achieve good control result without the accurate mathematical model of the subject. In short, the control method of thruster-AUV's motion adjustment based on fuzzy control is feasible and has a certain reference value.

\section{Acknowledgement}

This work was financially supported by the National 863 High Technology Development Plan Project (2011AA09A106) and China Postdoctoral Science Special Foundation (2012T50331).

\section{References}

[1] Akçakaya Halil. Robust control of variable speed autonomous underwater vehicle [J]. Advanced Robotics, 201428 (9) 601-611

[2] Sanitwong Na Ayutthaya Sarinya. Autonomous underwater vehicle for coastal survey [C]. Korea: International Society of Offshore and Polar Engineers, 2014.410-414

[3] Qin Xu-ping. Development and Prospect of Fuzzy Control Theory [J]. Control Engineering of China, 200512 137-139

[4] Li Shi-yong. Fuzzy Control Theory, 2011

[5] Huang Wei-hua. Analysis and Design of Membership Function for a Typical Fuzzy Controller [J]. Fuzzy Systems and Mathematics, 2010 24(5) 83-90

[6] Wang Lei. Fuzzy Control Theory and Applications, 1997 